\title{
First Physics Results of AWAKE, a Plasma Wakefield Acceleration Experiment at CERN
}

\author{
Patric Muggli, Allen Caldwell ${ }^{* \dagger}$ \\ Max Planck Institute for Physics \\ E-mail: muggli@mpp.mpg.de
}

\begin{abstract}
AWAKE is a plasma wakefield acceleration experiment using the $12 \mathrm{~cm}$-long, $400 \mathrm{GeV}$ proton bunch of the CERN SPS. In order to reach an acceleration gradient in the $\mathrm{GeV} / \mathrm{m}$ range, the plasma electron density is $7 \times 10^{14} \mathrm{~cm}^{-3}$. The transverse self-modulation instability (SMI), strongly seeded by an laser ionization front, turns the long bunch into a train of micro-bunches at the plasma wavelength scale $(\sim 1 \mathrm{~mm})$ that resonantly drives the wakefields to large amplitude. Low energy electrons $(\sim 15 \mathrm{MeV})$ can then be externally injected and accelerated to $\mathrm{GeV}$ energies. The plasma source is a laser-ionized rubidium vapor source. The vapor density is measured with $<0.5 \%$ accuracy at both ends of the source.

The detection of the SMI is based on diagnostics aimed at measuring the proton bunch modulation: fluorescent screens for measuring the proton bunch transverse density profile at two locations, optical transition radiation (OTR) and streak camera for direct observation of the modulation, and coherent transition radiation (CTR) for modulation frequency measurements.

The first experiments focus of the study of the SMI. Experimental results obtained in late 2016 show signs of self-modulation on all diagnostics. Further SMI experiments will be conducted in 2017, together with the installation of the RF-gun and of the electron spectrometer. Injection and acceleration experiments will be conducted in 2018.

After a general introduction to AWAKE and to its physics, the experimental apparatus will be briefly described and the most recent experimental results will be presented. Mid- and long-term plans, including future experiments, the development of scalable plasma sources and possible applications to HEP will be discussed.
\end{abstract}

The European Physical Society Conference on High Energy Physics

5-12 July

Venice, Italy

${ }^{*}$ Speaker.

${ }^{\dagger}$ for the AWAKE Collaboration 


\section{1. introduction}

Accelerators provide high-energy particles for physics discoveries. The general trend is towards higher energies, leading to larger and more expensive accelerators. There are therefore two quests for new technologies. One for higher field magnets for circular proton machines and one for higher accelerating gradients for linear electron/positron machines.

Plasma-based particle accelerators $[1,2]$ demonstrated very large accelerating gradients, exceeding $100 \mathrm{GeV} / \mathrm{m}$. Electrons have been accelerated from rest to $4.2 \mathrm{GeV}$ in a $9 \mathrm{~cm}$-long plasma driven by a petawatt power laser pulse [3], and from 42 to $84 \mathrm{GeV}$ in a $85 \mathrm{~cm}$-long plasma driven by a $42 \mathrm{GeV}$ electron bunch [4]. However, these drivers carry an energy of less than $100 \mathrm{~J}$, thereby limiting the acceleration length and the energy gain of the accelerated bunch to between 10 and $100 \mathrm{GeV}$. Reaching higher energies with those schemes requires staging of multiple plasmas. This adds significant complexity to the accelerator. In addition, the distance between each stage necessary to capture and re-focus the accelerated bunch into the next stage significantly decreases the average accelerating gradient [5].

Proton bunches carry much larger amounts of energy, from $20 \mathrm{~kJ}$ for those produced by the CERN SPS (Super Proton Synchrotron, $3 \times 10^{11} p^{+}, 400 \mathrm{GeV} / p^{+}$) to $112 \mathrm{~kJ}$ for those produced by the LHC (Large Hadron Collider $10^{11} p^{+}, 7 \mathrm{TeV} / p^{+}$). Bunches with such large energy could in principle drive a much longer plasma section and produce accelerated electron bunches of hundreds of GeVs in a single acceleration stage only a few hundred meters long [6].

Plasma-based particle accelerators operate at high frequencies, hundreds of $\mathrm{GHz}$ to multi- $\mathrm{THz}$, in order to reach high accelerating gradients. The field they can sustain is on the order of the wave breaking field $\mathrm{E}_{W B}=\mathrm{m}_{e} \mathrm{c} \omega_{p e} / \mathrm{e}, \mathrm{E}_{\max } \cong\left(n_{b} / n_{e}\right) E_{W B}$. Here $\mathrm{n}_{b}$ is the drive beam density, $\mathrm{n}_{e}$ is the plasma electron density and $\omega_{p e}=\left(n_{e} e^{2} / \varepsilon_{0} m_{e}\right)^{1 / 2}$ is the plasma electron angular frequency. To fit in the wakefield structure of typical size $\lambda_{p e}=2 \pi c / \omega_{p e}$, the bunch rms longitudinal and transverse dimensions must satisfy $k_{p e} \sigma_{z} \cong 1$ and $k_{p e} \sigma_{r} \cong 1$, respectively. Here $k_{p e}=2 \pi / \lambda_{p e}$ and we assume that the wakefields phase velocity given by the relativistic velocity is $v_{\phi}=v_{b} \cong c$.

\section{AWAKE}

AWAKE uses the long, $\sigma_{z} \cong 8-12 \mathrm{~cm}$, but tightly focused, $\sigma_{r} \cong 200 \mu \mathrm{m}$ proton bunch produced by the CERN SPS to drive wakefields in a $10 \mathrm{~m}$-long plasma [7]. Choosing the plasma density $\mathrm{n}_{e}=7 \times 10^{14} \mathrm{~cm}^{-3}$ to satisfy $\mathrm{k}_{p e} \sigma_{r}=1$ allows for $\mathrm{E}_{W B}$ to reach $2.5 \mathrm{GeV} / \mathrm{m}$. However, since with $3 \times 10^{11}$ protons the bunch density is only $\sim 5 \times 10^{-3} \mathrm{n}_{e}$ and $\mathrm{k}_{p e} \sigma_{z} \gg 1$, the proton bunch is very ineffective at driving wakefields. AWAKE therefore relies on a self-modulation process [8] to transform the long proton bunch into a train of short bunches separated by $\lambda_{p e}$, each also satisfying $\mathrm{k}_{p e} \sigma_{z} \leq 1$. The train of $\sim \sigma_{z} / \lambda_{p e} \cong 100$ bunches then resonantly drives wakefields to amplitudes comparable to $\mathrm{E}_{W B}$.

In AWAKE the self-modulation process is seeded by a short laser pulse propagating co-linearly with, and in the middle of the proton bunch. The laser pulse creates a sharp $\left(\ll \lambda_{p e}\right)$ ionization front and protons propagating in the plasma, behind the laser pulse, experience the seeded selfmodulation (SSM) process [9]. The SSM is a purely transverse effect that creates the train of short bunches through the periodic focusing and defocusing action of the wakefields. Seeding is 
essential. It fixes the phase of the wakefields with respect to the ionizing laser pulse, a necessary condition in order to deterministically inject electrons in the accelerating and focusing phase of the wakefields. Since in addition to growing along the plasma, the wakefields grow along the bunch, the electrons are injected approximately 100 wakefields periods behind the laser pulse. This puts a stringent constraint on the relative uniformity of the plasma density along the plasma, on the order of $\frac{1}{4} \frac{\sigma_{z}}{\lambda_{p e}}$ or $0.2 \%$ in the AWAKE case. The factor $\frac{1}{4}$ comes from the fact that, in the linear regime, only this fraction of the wakefields period is accelerating and focusing for the electron bunch.

Initial experimental results show clear evidence of the occurrence of the SSM process. At present, the diagnostics are only characterizing the proton bunch.

\section{Diagnostics}

The first diagnostic uses two screens located 2 and $10 \mathrm{~m}$ downstream from the $10 \mathrm{~m}$-long plasma exit [10] to observe the effect of the SSM on the (time integrated) transverse profile of the proton bunch. Important information about the SSM process can in principle be extracted from the angle and radial position of the defocused protons at the screens [11]: The point along the plasma where the defocused protons leave the wakefields; The transverse momentum gained by the protons from the wakefields; And from these two values, an estimate of the wakefields amplitude and the growth rate of the SSM.

The second diagnostic time-resolves the (incoherent) optical transition radiation (OTR) emitted by the protons when traversing a thin metallic screen also placed downstream from the plasma. The streak camera must have a time resolution at the picosecond level to measure the $90-270 \mathrm{GHz}$ modulation frequencies expected from operation at plasma densities between 1 and $10 \times 10^{14} \mathrm{~cm}^{-3}$. At low plasma densities $\left(<3 \times 10^{14} \mathrm{~cm}^{-3}\right)$ the short bunches are directly observable on the streak camera images, i.e., in time. At higher densities the modulation frequency is obtained from the power spectrum of the image profile fast Fourier transform [12].

The third diagnostic is based on the coherent spectrum of the transition radiation (CTR) emitted when the train of bunches traverses a second metallic foil. The CTR spectrum consists essentially of a contribution at low frequency $\left(<\mathrm{c} / \sigma_{z} \sim \mathrm{GHz}\right)$ corresponding to the bunch envelope and of a peak corresponding to the modulation frequency $(>90 \mathrm{GHz})$. Detecting the presence of harmonics of the modulation frequency in the CTR spectrum would signal the formation of microbunches shorter than half the modulation wavelength, i.e., of the non-linear development of the SSM process. More harmonics content indicates shorter bunches. The CTR frequency is coarsely analyzed using Schottky diodes with high-pass cut-off waveguide filters at 90, 170 and $220 \mathrm{GHz}$. The frequency of the CTR is also finely analyzed $(\Delta \mathrm{f}<1-2 \mathrm{GHz})$ using various heterodyne frequency mixing systems [13].

\section{Some Key Measurements}

The first key measurement is the demonstration of the occurrence of the SSM process using all three diagnostics. This includes the measurement of the modulation frequency and its comparison with the expected plasma frequency.

The second one is the demonstration of the seeding of the self-modulation process. 
The third one is the quantification of the (lack of) sensitivity of the SSM to variations of the initial proton bunch parameters. This is achieved by sending a low energy replica of the ionizing laser pulse to the streak camera at the same time as the OTR light from the proton bunch. Since this replica is synchronized ( $\ll 1 \mathrm{ps}$ jitter) with the main laser pulse, it can be precisely delayed and used to observe fine time variations of the micro-bunches structure in short time windows ( $73 \mathrm{ps)}$ delayed by up to a few hundred picoseconds with respect to the main pulse. Since the wakefields are driven by, and tied to the bunch train, reproducibility of the micro-bunches in timing with respect to the main laser pulse is an indication of the reproducibility of the wakefields phase with respect to the same laser pulse and seed point. This of course essential for deterministic injection of electrons in the accelerating and focusing phase of the wakefields, approximately one hundred periods behind their seed point.

Other experiments include: comparison between SSM process and unseeded self-modulation, by placing the ionizing laser pulse some time ahead of the proton bunch; Observation and characterization of the hose instability and its competition with the SSM [14]; Detailled study of the SSM along the proton bunch using the electron bunch injected with various delays with respect to the seed point.

\section{Prospects}

Ongoing experiments focus on SSM physics. At the same time, an electron RF-injector is in the installation and commissioning phases. Acceleration experiments will occur in 2018. These will aim to sample the wakefields with a low energy $(\sim 5-20 \mathrm{MeV})$ electron bunch with length comparable to the wakefields period. Later experiments, planned for 2021 and beyond, will aim to accelerate an electron bunch that is short when compared to the wakefields period and demonstrate multi-GeV energy gain with a narrow (\%-level) energy spread while preserving the incoming bunch emittance. Simulation results show that when loading the wakefields one can also blow-out the plasma electrons remaining in the accelerating structure and reach a focusing field structure that maintains the emittance of most of the charge of the witness bunch [15]. Possible application of the acceleration scheme for electron/fixed target as well as electron/proton collision experiments are also explored [16].

\section{References}

[1] T. Tajima and J. Dawson, 1979, Phys. Rev. Lett., 43, 267

[2] P. Chen et al., 1985, Phys. Rev. Lett., 54, 693

[3] W. Leemans et al., 2014, Phys. Rev. Lett., 113, 245002

[4] I. Blumenfeld et al., 2017, Nature 445, 741

[5] C.A. Lindstrøm et al.,2016, Nucl. Instr. and Meth. in Phys. Res. A, 829, 224

[6] A. Caldwell et al., 2009, Nature Physics, 5, 363

[7] AWAKE Collaboration, 2014, Plasma Phys. Control. Fusion, 56, 084013 E. Gschwendtner et al., 2016, Nucl. Instr. and Meth. in Phys. Res. A, 829, 76 A. Caldwell et al., 2016, Nucl. Instr. and Meth. in Phys. Res. A, 829, 3 
[8] N. Kumar et al., 2010, Phys. Rev. Lett., 104, 255003

[9] P. Muggli et al., Plasma Physics and Controlled Fusion, 60(1) 014046 (2017), arXiv:1708.01087

[10] E. Öz et al. 2014 Nucl. Instr. and Meth. in Phys. Res. A, 740(11), 197, E. Öz et al. 2016 Nucl. Instr. and Meth. in Phys. Res. A, 829, 321

[11] M. Turner et al., 2017 Nucl. Instrum. Meth. A, 854, 100

[12] K. Rieger et al., 2017, Review of Scientific Instruments, 88, 025110

[13] F. Braunmueller et al., 2018, accepted for publication in Nucl. Instr. and Meth. in Res. Phys. A

[14] J. Vieira et al., 2014, Phys. Rev. Lett., 112, 205001

[15] V. K. Berglyd Olsen et al., in preparation

[16] A. Caldwell and M. Wing, 2016, Eur. Phys. J. C, 76, 463 